\title{
Performance Evaluation in Single or Multi-Cluster C-RAN Supporting Quasi-Random Traffic
}

\author{
Iskanter-Alexandros Chousainov, Ioannis Moscholios, Alexandros Kaloxylos, Senior Member, IEEE, \\ and Michael Logothetis, Senior Member, IEEE
}

\begin{abstract}
In this paper, a cloud radio access network (C-RAN) is considered where the remote radio heads (RRHs) are separated from the baseband units (BBUs). The RRHs in the C-RAN are grouped in different clusters according to their capacity while the BBUs form a centralized pool of computational resource units. Each RRH services a finite number of mobile users, i.e., the call arrival process is the quasi-random process. A new call of a single service-class requires a radio and a computational resource unit in order to be accepted in the C-RAN for a generally distributed service time. If these resource units are unavailable, then the call is blocked and lost. To analyze the multi-cluster C-RAN, we model it as a single-rate loss system, show that a product form solution exists for the steady state probabilities and propose a convolution algorithm for the accurate determination of congestion probabilities. The accuracy of this algorithm is verified via simulation. The proposed model generalizes our recent model where the RRHs in the C-RAN are grouped in a single cluster and each RRH accommodates quasi-random traffic.
\end{abstract}

Index Terms-cloud, radio access, call blocking, product form, quasi-random.

\section{INTRODUCTION}

$\mathrm{T}$ E cloud radio access network (C-RAN) is considered as a promising fifth generation $(5 \mathrm{G})$ network architecture which is anticipated to address the increasing demand for bandwidth-hungry applications, decreased latency (in the order of a few ms), improved data rate (up to $1 \mathrm{Gbps}$ ) and consequently the rapid growth of wireless traffic which is expected to reach 110 ExaBytes/month by the end of 2023 [1], [2].

The C-RAN architecture includes a large number of base stations where the remote radio heads (RRHs) are separated from the baseband units (BBUs) [3]. The BBUs form a centralized pool of computational resource units (RUs) which is connected to the RRHs via the common public radio interface (CPRI) with a high-capacity fronthaul [4].

Manuscript received February 24, 2020; revised March 31, 2020. Date of publication May 11, 2020. Date of current version May 11, 2020. The associate editor prof. Pascal Lorenz has been coordinating the review of this manuscript and approved it for publication.

The part of this paper was presented at the International Conference on Software, Telecommunications and Computer Networks (SoftCOM) 2019.

I-A. Chousainov, I. Moscholios and A. Kaloxylos are with the Department of Informatics and Telecommunications, University of Peloponnese, 22100 Tripolis, Greece, e-mail: (ichousain@uop.gr, idm@uop.gr and kaloxyl@uop.gr).

M. Logothetis is with the WCL, Department of Electrical and Computer Engineering, University of Patras, 26504 Patra, Greece, email: (mlogo@upatras.gr)

Digital Object Identifier (DOI): 10.24138/jcomss.v16i2.1013
To benefit from network function virtualization, we consider virtualized BBU resources (V-BBU) [3] where the BBU functionality and services have been virtualized in the form of virtual network functions (VNFs) [5]. We focus on the radio resource management, a BBU function that can be virtualized as a VNF, which is responsible not only for the radio resource allocation but also for the call admission control (CAC) of mobile users (MUs).

A CAC is a significant quality of service (QoS) mechanism that gives access to the RUs required by MUs and provides fairness among different mobile services/applications. Considering call-level traffic in the C-RAN, such a QoS mechanism is a bandwidth (resource) sharing policy since it affects call blocking probabilities (CBP). Generally speaking, it is desirable to have efficient recursive formulas or convolution algorithms for the determination of CBP. Such formulas or algorithms can be helpful in network planning and can be obtained via teletraffic loss/queueing models [6], [7]. In this paper, we focus on convolution algorithms which are adopted in the literature not only in the case of loss/queueing models whose steady state probabilities have a product form solution (PFS) but also in the case of non-PFS models (e.g., [8][16]). Such algorithms are advantageous not only due to their low computational complexity but also because they can incorporate various resource sharing policies such as the complete sharing policy, the bandwidth reservation policy and threshold-based policies (e.g., [17]-[24]).

On the one hand, various aspects of the C-RAN architecture have been investigated and analyzed the last few years, such as the capacity demands and possible functional splits on the fronthaul network [25], [26], energy and cost saving issues [27], [28], security challenges [29], resource allocation issues related to RRH selection, spectrum management and throughput maximization [30], as well as the dimensioning problem of the necessary number of V-BBU required to handle a specific number of RRHs [31], [32]. The latter focus only on the V-BBU and model them as a queueing system in which the arrival process of jobs follows a batched Poisson process and the service time is exponentially distributed. On the other hand, there are very few papers that consider CAC in the C-RAN and provide analytical formulas or algorithms for the CBP determination ([33]-[35], [37]-[39]).

In [33], all RRHs form a single cluster in the C-RAN. The latter accommodates calls of a single service-class that arrive in the system according to a Poisson process. A new call 
requires a computational RU from the $\mathrm{V}-\mathrm{BBU}$ and a radio RU from the serving RRH. If both RUs are available, then the new call is accepted in the serving RRH and remains in the system for a generally distributed service time. Otherwise, call blocking occurs. The analytical model of [33], named single-class-single-cluster (SC-SC) model, is based on a multidimensional reversible Markov chain which leads to a PFS for the steady-state probabilities. The PFS leads to an accurate CBP calculation either via the complex procedure of enumeration and processing of the system's state space or via recursive formulas. In [34], [35], the SC-SC model is extended to include the case of overlapping cells via the notion of a direct routing network [36]. In [37], a convolution algorithm is proposed for the CBP determination of the SC-SC model. In [38], the SC-SC model is extended to include the case where the RRHs can be grouped in multi-clusters (SC-MC model). In that case, the RRHs that belong to a cluster have the same amount of radio RUs. Recently, the authors have extended the SC-SC model to include the case of a quasi-random call arrival process [39]. This process appears when the RRHs serve calls generated by a finite number of MUs. In that sense, the quasirandom process is smoother than the Poisson process (where calls are generated by an infinite number of users) [7]. We name the model of [39], finite SC-SC (f-SC-SC) model.

In this paper, we focus on the models of [38] and [39]. More precisely, we extend the SC-MC model of [38] by incorporating the quasi-random call arrival process. The proposed model, named finite SC-MC (f-SC-MC) model, generalizes the f-SC-SC model of [39], since it covers the more complicated multi-cluster case. Our contribution is three-fold: 1) we review the SC-MC model, provide some corrections for the CBP formulas (presented in [38]) which are necessary for their implementation and propose a convolution algorithm for the CBP calculation, 2) we propose the f-SC-MC model, analyze it via a multidimensional reversible Markov chain and show that a PFS exists for the steady-state probability distribution and 3 ) we propose a convolution algorithm (which is based on the proposed PFS) for the exact determination of the congestion probabilities in the f-SC-MC model and compare the results with those obtained via the SC-SC model of [33], the SC-MC model of [38] and the f-SC-SC model of [39]. Simulation results verify the accuracy of all models.

The remainder of this paper is as follows: In Section II, we review the SC-MC model and propose a convolution algorithm for the calculation of CBP. In Section III, we present the proposed f-SC-MC model and a 3-step convolution algorithm for the determination of the various performance measures. In Section IV, we present analytical and simulation results for the congestion probabilities of the f-SC-SC and the fSC-MC models and compare them with the corresponding analytical results of [33] and [38], respectively. We conclude in Section V.

\section{REVIEW OF THE SC-MC MODEL}

\section{A. The Analytical Model}

Consider the C-RAN of Fig. 1 where the RRHs are separated from the V-BBU. There exist $Z$ different classes of
RRHs. Each class $z(z=1, \ldots, Z)$ forms a cluster of $M_{z}$ RRHs. A RRH that belongs to class $z$ has $C_{z}$ radio RUs which are allocated to the serving calls of the MUs. The V-BBU has a capacity of $T$ computational RUs.

Similar to the SC-SC model of [33], new calls arrive in the C-RAN according to a Poisson process. Let $\lambda_{z}$ be the arrival rate of new calls in a serving RRH of class $z$. The call admission mechanism is as follows: a new call requires a computational RU from the $\mathrm{V}-\mathrm{BBU}$ and a radio RU from the serving RRH of class $z$. If both RUs are available, then the new call is accepted in the serving RRH for a generally distributed service time with mean $\mu^{-1}$. Otherwise, the call is blocked and lost.

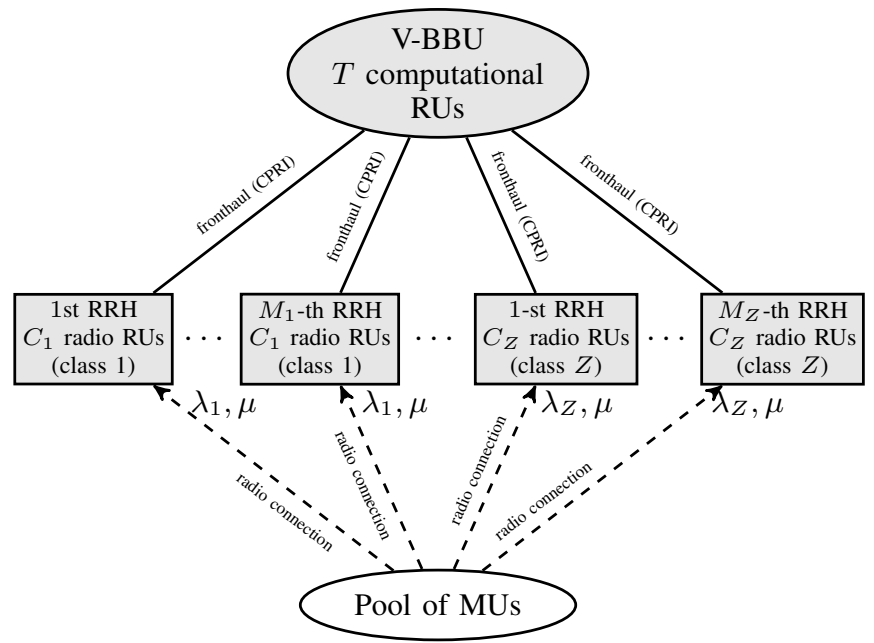

Fig. 1. The SC-MC model.

Let $n_{z, m}$ be the number of in-service calls in the $m$-th RRH that belongs to class $z\left(z=1, \ldots, Z\right.$ and $\left.m=1, \ldots, M_{z}\right)$. Then, the number of calls serviced in all RRHs of all $Z$ classes is expressed by the steady-state vector $\boldsymbol{n}=\left(n_{1,1}, \ldots\right.$, $\left.n_{1, M_{1}}, \ldots, n_{z, 1}, \ldots, n_{z, M_{z}}, \ldots, n_{Z, 1}, \ldots, n_{Z, M_{Z}}\right)$ and the corresponding steady-state probability distribution, $P(\boldsymbol{n})$ has the following PFS [38]:

$$
P(\boldsymbol{n})=G^{-1}\left(\prod_{z=1}^{Z} \prod_{m=1}^{M_{z}} \frac{\alpha_{z}^{n_{z, m}}}{n_{z, m} !}\right)
$$

where: $\alpha_{z}=\lambda_{z} / \mu$ is the offered traffic-load (in erl) in every RRH that belongs to class $z, G \equiv G(\boldsymbol{\Omega})=$ $\sum_{\boldsymbol{n} \in \boldsymbol{\Omega}} \prod_{z=1}^{Z} \prod_{m=1}^{M_{z}} \alpha_{z}^{n_{z, m}} / n_{z, m}$ ! and $\boldsymbol{\Omega}$ is the system's state space given by $\boldsymbol{\Omega}=\left\{\boldsymbol{n}: 0 \leq n_{z, 1}, \ldots, n_{z, M_{z}} \leq C_{z}, 0 \leq\right.$ $\left.\sum_{z=1}^{Z} \sum_{m=1}^{M_{z}} n_{z, m} \leq T\right\}$.

Based on $P(\boldsymbol{n})$, we can determine the total CBP, $B_{z, t o t}$, of calls serviced in a RRH of class $z$ via:

$$
B_{z, t o t}=B_{z, r}+B_{c}
$$

where: $B_{z, r}, B_{c}$ refer to the blocking caused due to insufficient radio and computational RUs, respectively.

The values of $B_{z, t o t}$ can be computed either via a brute-force evaluation method (see Section II-B) or via a recursive method (see Section II-C) proposed in [38] or via a convolution algorithm (see Section II-D) proposed herein. 


\section{B. The Brute Force Evaluation Method}

The values of $B_{z, r}$ can be computed via (1) as follows:

$B_{z, r}=\frac{1}{G}\left[\frac{\alpha_{z}^{C_{z}}}{C_{z} !} \sum_{n \in \boldsymbol{\Omega}_{<T}^{z, 1, C_{z}}}\left(\prod_{\substack{w=1, w \neq z}}^{Z} \prod_{m=1}^{M_{w}} \frac{\alpha_{w}^{n_{w, m}}}{n_{w, m} !}\right)\left(\prod_{m=2}^{M_{z}} \frac{\alpha_{z}^{n_{z, m}}}{n_{z, m} !}\right)\right]$

where: $\boldsymbol{\Omega}_{<T}^{z, 1, C_{z}}=\left\{\boldsymbol{\Omega}^{z, 1, C_{z}} \cap \boldsymbol{\Omega}_{<T}\right\}, \boldsymbol{\Omega}^{z, 1, C_{z}}=\left\{\boldsymbol{n}: n_{z, 1}=\right.$ $\left.C_{z}\right\}, \boldsymbol{\Omega}_{<T}=\left\{\boldsymbol{n}: \sum_{z=1}^{Z} \sum_{m=1}^{M z} n_{z, m}<T\right\}$.

Equation (3) gives the values of $B_{z, r}$ for the 1st RRH of class $z$. However, since we have assumed that the RRHs of class $z$ are identical and have the same offered traffic-load $\alpha_{z}$ and the same capacities $C_{z}$, then (3) refers to the blocking caused due to insufficient radio RUs in any RRH of class $z$.

Similarly, by denoting as $\boldsymbol{\Omega}_{=T}=\left\{\boldsymbol{n}: \sum_{z=1}^{Z} \sum_{m=1}^{M z} n_{z, m}=\right.$ $T\}$, the values of $B_{c}$ are given by:

$$
B_{c}=\sum_{\boldsymbol{n} \in \boldsymbol{\Omega}_{=T}} P(\boldsymbol{n})
$$

\section{The Recursive Evaluation Method}

The determination $B_{z, r}$ and $B_{c}$ according to the recursive method of [38], requires the following functions:

$$
\begin{aligned}
& C_{R}(T, \boldsymbol{M})=\sum_{\boldsymbol{n} \in \boldsymbol{\Omega}_{=}} \prod_{z=1}^{Z} \prod_{m=1}^{M_{z}} \frac{\alpha_{z}^{n_{z, m}}}{n_{z, m} !} \\
& R_{R}(T, \boldsymbol{M})=\sum_{\boldsymbol{n} \in \boldsymbol{\Omega}_{<T}} \prod_{z=1}^{Z} \prod_{m=1}^{M_{z}} \frac{\alpha_{z}^{n_{z, m}}}{n_{z, m} !}
\end{aligned}
$$

where: $\boldsymbol{M}=\left(M_{1}, \ldots, M_{z}, \ldots, M_{Z}\right)^{T}$ is a column vector of length $Z$.

The recursive calculation of $C_{R}(T, M)$ and $R_{R}(T, M)$ is based on (7) and (8), including some necessary corrections (for their software implementation) compared to the formulas that appear in [38]:

$$
C_{R}(T, \boldsymbol{M})= \begin{cases}\alpha_{z}^{X_{2, z}} / X_{2, z} !, & \text { for } \boldsymbol{M}=\boldsymbol{e}_{z} \\ \sum_{i=X_{1, z}}^{X_{2, z}} \frac{\alpha_{z}^{i}}{i !} C_{R}\left(T-i, \boldsymbol{M}-\boldsymbol{e}_{z}\right), & \text { for } \boldsymbol{M}>\boldsymbol{e}_{z}\end{cases}
$$

where: $\boldsymbol{e}_{z}=(0, \ldots, 0,1,0, \ldots, 0)^{T}$ is a column vector of length $Z$ with 1 in element $z$ and 0 elsewhere, while $X_{1, z}=\max \left[0, T-\left(M_{z}-1\right) C_{z}-\sum_{w \neq z} M_{w} C_{w}\right]$ and $X_{2, z}=$ $\min \left[C_{z}, T\right]$.

$R_{R}(T, M)= \begin{cases}1, & \text { for } T=1 \\ R_{R}(T+1, M)-C_{R}(T, M), & \text { for } 2 \leq T \leq \sum_{z=1}^{Z} M_{z} C_{z} \\ \prod_{z=1}^{Z}\left(\sum_{i=0}^{C_{z}} \frac{\alpha_{z}^{i}}{i !}\right)^{M_{i}}, & \text { for } T=\sum_{z=1}^{Z} M_{z} C_{z}+1\end{cases}$

Based on (7), (8), the values of $B_{z, r}$ and $B_{c}$ can be computed via (9) and (10) respectively:

$$
\begin{gathered}
B_{z, r}=\frac{\alpha_{z}^{C_{z}}}{C_{z} !} \frac{R_{R}\left(T-C_{z}, \boldsymbol{M}-\boldsymbol{e}_{z}\right)}{R_{R}(T+1, \boldsymbol{M})} \\
B_{c}=\frac{C_{R}(T, \boldsymbol{M})}{R_{R}(T+1, \boldsymbol{M})}
\end{gathered}
$$

Regarding the computational complexity of the previous recursive method, it is proved that it is upper bounded by the term $\left(\left(\max _{z} C_{z}\right)^{2}+\max _{z} C_{z}\right) M^{2}$. The latter is based on the assumption that $T^{z} \leq \sum_{z=1}^{Z} M_{z} C_{z}$. In the case of $T>\sum_{z=1}^{Z} M_{z} C_{z}$, which results in an overprovision of the computational RUs, a large pool approximation is proposed for the determination of blocking probabilities.

The previous method leads to an efficient way for the computation of $B_{z, r}$ and $B_{c}$ and consequently $B_{z, t o t}$ (via (2)). However, it is not clear how: i) the calculation of the occupancy distribution of the computational RUs will be achieved and ii) this method can be extended when non-Poisson arrival processes should be studied. Finally, note that in the particular case of $T>\sum_{z=1}^{Z} M_{z} C_{z}$, the model behaves as $M$ individual Erlang B loss systems of capacity $C_{z}(z=1, \ldots, Z)$ radio RUs and offered traffic-load equal to $\alpha_{z}$. In that case, $B_{c}=0$ and $B_{z, r}=E_{C_{z}}\left(\alpha_{z}\right)$ where $E_{C_{z}}\left(\alpha_{z}\right)$ is the Erlang B formula. The recursive evaluation method of (8) and (9) is not defined when $T>\sum_{z=1}^{Z} M_{z} C_{z}$. The previous drawbacks can be dealt with the proposed convolution algorithm that follows.

\section{The Proposed Convolution Algorithm}

In order to efficiently determine $B_{z, t o t}$ as well as the occupancy distribution of the computational RUs, we propose the following convolution algorithm:

Step 1

For each class $z$ of RRHs $(z=1, \ldots, Z)$ determine the occupancy distribution of each of the $M_{z}$ RRHs, $q_{z, m}(j)$, where $m=1, \ldots, M_{z}$ and $j=1, \ldots, C_{z}$, via:

$$
q_{z, m}(j)= \begin{cases}\frac{\alpha_{z}^{j}}{j !} q_{z, m}(0), & \text { for } j=1, \ldots, C_{z} \\ 0, & \text { for } j=C_{z}+1, \ldots, T\end{cases}
$$

The values of $q_{z, m}(j)$ can be normalized via the constant $G_{z, m}=\sum_{j=0}^{C_{z}} q_{z, m}(j)$ and are denoted via $q_{z, m}^{\prime}(j)=$ $q_{z, m}(j) / G_{z, m}$.

Step 2

Following the successive convolution of all RRHs (of all classes $Z)$, apart from the $(z, 1) \mathrm{RRH}$, determine the aggregated occupancy distribution:

$$
\begin{aligned}
& Q_{(-(z, 1))}= \\
& =q_{1,1}^{\prime} * \ldots * q_{1, M_{1}}^{\prime} * \ldots * q_{z, 2}^{\prime} * \ldots * q_{z, M_{z}}^{\prime} * \ldots * q_{Z, 1}^{\prime} * \ldots * q_{Z, M_{Z}}^{\prime}
\end{aligned}
$$

The convolution operation between two occupancy distributions $q_{z, u}^{\prime}$ and $q_{z, w}^{\prime}$ is given by:

$$
q_{z, u}^{\prime} * q_{z, w}^{\prime}=\left\{\begin{array}{l}
q_{z, u}^{\prime}(0) \cdot q_{z, w}^{\prime}(0), \sum_{x=0}^{1} q_{z, u}^{\prime}(x) \cdot q_{z, w}^{\prime}(1-x), \\
\ldots, \sum_{x=0}^{T} q_{z, u}^{\prime}(x) \cdot q_{z, w}^{\prime}(T-x)
\end{array}\right\}
$$

Since this convolution may not lead to a normalized distribution, it is recommended to normalize the results of (12) via the normalization constant, $G_{z, u, w}$. 
Step 3

Compute $B_{z, t o t}$ based on the normalized values obtained from the convolution operation of step 2, as follows:

$$
B_{z, t o t}=B_{z, r}+B_{c}=\frac{1}{G}\left(q_{z, 1}^{\prime}\left(C_{z}\right) \sum_{r=0}^{T-1-C_{z}} Q_{(-(z, 1))}^{\prime}(r)+q(T)\right)
$$

where: $q_{z, 1}^{\prime}\left(C_{z}\right)$ refers to the case of unavailable radio RUs in the $(z, 1) \mathrm{RRH}$ (already determined in step 1$), Q_{(-(z, 1))}^{\prime}(r)$ are the normalized values of $Q_{(-(z, 1))}(r)$, while $q(T)$ refers to the un-normalized probability of unavailable computational RUs, given by $q(T)=\sum_{r=0}^{T} Q_{(-(z, 1))}^{\prime}(r) \cdot q_{z, 1}^{\prime}(T-r)$ and $G$ is the normalization constant of the final convolution operation $Q_{(-(z, 1))}^{\prime} * q_{z, 1}^{\prime}$ determined via (13).

The notation of (14) leads to the values of $B_{z, t o t}$ for the 1 st RRH of class $z$. Since we have already assumed that all RRHs of the same class $z$ have the same capacity $C_{z}$ and the same offered traffic-load $\alpha_{z}$, it is obvious that (14) refers to the $B_{z, t o t}$ of any RRH of the same class $z$.

Based on the convolution algorithm, we can obtain the occupancy distribution of the computational RUs, as follows:

$$
\begin{array}{ll}
q^{\prime}(0)=Q_{(-(z, 1))}^{\prime}(0) \cdot q_{z, 1}^{\prime}(0) / G, & j=0 \\
q^{\prime}(j)=\sum_{r=0}^{j} Q_{(-(z, 1))}(r)^{\prime} \cdot q_{z, 1}^{\prime}(j-r) / G, & j=1, \ldots, T
\end{array}
$$

where: $G$ is the normalization constant of the final convolution operation $Q_{(-(z, 1))}^{\prime} * q_{z, 1}^{\prime}$.

Regarding the computational complexity of (13) for the SCMC model, it is in the order of $O\left(T^{2}\right)$ while the corresponding complexity in order to obtain the values of (15) is in the order of $O\left(M T^{2}\right)$.

Note that when $Z=1$, then all RRHs form a single cluster in the C-RAN and we have the SC-SC model. In that case, the interested reader may resort to [33] and [39] for the analysis of the brute force evaluation and the recursive evaluation methods of this model. Herein, we only present the corresponding 3step convolution algorithm for the CBP calculation [39]:

Step 1

For each of the $M$ RRHs of the C-RAN, determine the occupancy distribution $q_{m}(j)$, where $m=1, \ldots, M$ and $j=1, \ldots, C$, via:

$$
q_{m}(j)= \begin{cases}\frac{\alpha^{j}}{j !} q_{m}(0), & \text { for } j=1, \ldots, C \\ 0, & \text { for } j=C+1, \ldots, T\end{cases}
$$

The normalized values of $q_{m}(j)$ are expressed as $q_{m}^{\prime}(j)=$ $q_{m}(j) / G_{m}$ where $G_{m}=\sum_{j=0}^{C} q_{m}(j)$.

Step 2

Following the convolution of all RRHs, apart from the 1st RRH, determine the aggregated occupancy distribution:

$$
Q_{(-1)}=q_{2}^{\prime} * \ldots * q_{m}^{\prime} * \ldots * q_{M}^{\prime}
$$

where the convolution of $q_{u}^{\prime}$ and $q_{w}^{\prime}$ is given by (13).

Step 3

Calculate the values of the total CBP, $B_{\text {tot }}$, according to the normalized values obtained in step 2 , as follows:

$$
B_{\text {tot }}=B_{r}+B_{c}=G^{-1}\left(q_{1}^{\prime}(C) \sum_{r=0}^{T-C-1} Q_{(-1)}(r)+q(T)\right)
$$

where: $q_{1}^{\prime}(C)$ expresses the case where there are no radio RUs available in the 1st RRH while $q(T)$ expresses the (un-normalized) probability that all computational RUs are unavailable, given by $q(T)=\sum_{x=0}^{T} Q_{(-1)}(x) \cdot q_{1}^{\prime}(T-x)$ and $G$ is the normalization constant of the final convolution operation $Q_{(-1)} * q_{1}^{\prime}$ given via (13).

To determine the occupancy distribution of the computational RUs, we can use the formulas:

$$
\begin{array}{ll}
q^{\prime}(0)=Q_{(-1)}(0) \cdot q_{1}^{\prime}(0) / G, & j=0 \\
q^{\prime}(j)=\sum_{z=0}^{j} Q_{(-1)}(z) \cdot q_{1}^{\prime}(j-z) / G, & j=1, \ldots, T
\end{array}
$$

where: $G$ is the normalization constant of the final convolution operation $Q_{(-1)} * q_{1}^{\prime}$.

\section{The Proposed Finite SC-MC Model}

\section{A. The Analytical Model}

In the proposed $\mathrm{f}-\mathrm{SC}-\mathrm{MC}$ model, calls arrive in the $m$-th RRH that belongs to class $z(z=1, \ldots, Z$ and $m=1, \ldots$, $M_{z}$ ), according to a quasi-random process with mean arrival rate $\lambda_{z, m}=\left(N_{z, m}-n_{z, m}\right) v_{z, m}$, where $N_{z, m}$ is the finite population of MUs that can be served by the $m$-th RRH of class $z, n_{z, m}$ is the number of in-service calls in the $m$-th RRH of class $z$ and $v_{z, m}$ is the mean call arrival rate per idle MU in the $m$-th RRH of class $z$. The offered traffic-load per idle MU in the $m$-th RRH of class $z$, is $\alpha_{z, m \text {,idle }}=v_{z, m} / \mu$. Note that if $N_{z, m} \rightarrow \infty$, for $z=1, \ldots, Z$ and $m=1, \ldots, M_{z}$ and the total offered traffic-load remains constant, then the call arrival process becomes Poisson. In that case, the SC-MC model results. Similar to the SC-MC model an arriving call requires a radio $\mathrm{RU}$ from the serving $\mathrm{RRH}$ and a computational RU from the V-BBU. If these RUs are available then the new call is accepted in the serving RRH and remains for a generally distributed service time with mean $\mu^{-1}$. Otherwise, call blocking occurs.

Let the number of in-service calls in all RRHs be described by the steady-state vector $\boldsymbol{n}=\left(n_{1,1}, \ldots, n_{1, M_{1}}, \ldots, n_{z, 1}, \ldots\right.$, $\left.n_{z, M_{z}}, \ldots, n_{Z, 1}, \ldots, n_{Z, M_{Z}}\right)$. Also denote the steady-state vectors $\boldsymbol{n}_{z, m}^{+}=\left(n_{1,1}, \ldots, n_{1, M_{1}}, \ldots, n_{z, 1}, \ldots, n_{z, m}+1, \ldots\right.$, $\left.n_{z, M_{z}}, \ldots, n_{Z, 1}, \ldots, n_{Z, M_{Z}}\right), \boldsymbol{n}_{z, m}^{-}=\left(n_{1,1}, \ldots, n_{1, M_{1}}, \ldots\right.$, $\left.n_{z, 1}, \ldots, n_{z, m}-1, \ldots, n_{z, M_{z}}, \ldots, n_{Z, 1}, \ldots, n_{Z, M_{Z}}\right)$ and let $P_{\text {fin }}(\boldsymbol{n}), P_{\text {fin }}\left(\boldsymbol{n}_{z, m}^{-}\right), P_{\text {fin }}\left(\boldsymbol{n}_{z, m}^{+}\right)$be the probability distributions of states $\boldsymbol{n}, \boldsymbol{n}_{z, m}^{-}$and $\boldsymbol{n}_{z, m}^{+}$, respectively. Assuming that states $\boldsymbol{n}_{z, m}^{-}, \boldsymbol{n}, \boldsymbol{n}_{z, m}^{+}$belong to the state space $\boldsymbol{\Omega}$, we present in Fig. 2 the state transition diagram for the $m$-th RRH of the class $z$ of the proposed model.

Based on Fig. 2 and since the corresponding Markov chain is reversible, we can write the following local balance equations for states: a) $\boldsymbol{n}_{z, m}^{-}$and $\boldsymbol{n}$ (see (20)) and b) $\boldsymbol{n}$ and $\boldsymbol{n}_{z, m}^{+}($see $(21))$ where $z=1, \ldots, Z$ and $m=1, \ldots, M_{z}$ :

$$
\begin{gathered}
\left(N_{z, m}-n_{z, m}+1\right) v_{z, m} P_{\text {fin }}\left(\boldsymbol{n}_{z, m}^{-}\right)=n_{z, m} \mu P_{\text {fin }}(\boldsymbol{n}) \\
\left(N_{z, m}-n_{z, m}\right) v_{z, m} P_{\text {fin }}(\boldsymbol{n})=\left(n_{z, m}+1\right) \mu P_{\text {fin }}\left(\boldsymbol{n}_{z, m}^{-}\right)
\end{gathered}
$$




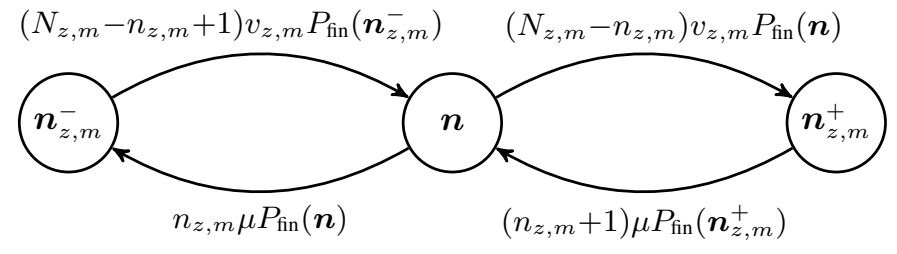

Fig. 2. State transition diagram for the $m$-th RRH of class $z$ of the f-SC-MC model.

The system of local balance equations is satisfied by the following PFS for $z=1, \ldots, Z, m=1, \ldots, M_{z}$ and $\boldsymbol{n} \in \boldsymbol{\Omega}$ :

$$
P_{\text {fin }}(\boldsymbol{n})=G^{-1}\left(\prod_{z=1}^{Z} \prod_{m=1}^{M_{z}}\left(\begin{array}{c}
N_{z, m} \\
n_{z, m}
\end{array}\right) \alpha_{z, m, \text { idle }}^{n_{z, m}}\right)
$$

where: $G \equiv G(\boldsymbol{\Omega})=\sum_{\boldsymbol{n} \in \boldsymbol{\Omega}} \prod_{z=1}^{Z} \prod_{m=1}^{M_{z}}\left(\begin{array}{c}N_{z, m} \\ n_{z, m}\end{array}\right) \alpha_{z, m, \text { idle }}^{n_{z, m}}$ and $\boldsymbol{\Omega}$ is the set of all possible states given by $\boldsymbol{\Omega}=\{\boldsymbol{n}: 0 \leq$ $\left.n_{z, 1}, \ldots, n_{z, M_{z}} \leq C_{z}, 0 \leq \sum_{z=1}^{Z} \sum_{m=1}^{M_{z}} n_{z, m} \leq T\right\}$.

In a system that services quasi-random traffic, we distinguish CBP in call congestion (CC) and time congestion (TC) probabilities. The latter refer to the proportion of time the system is congested. On the other hand, $\mathrm{CC}$ probabilities coincide with CBP. CC probabilities are only slightly lower than TC probabilities in quasi-random loss models, especially if the number of traffic sources is high [7]. Assuming Poisson arrivals (i.e., the call arrival process adopted in the SC-MC model), TC and CC probabilities coincide (PASTA property, [7]).

\section{B. The Proposed Convolution Algorithm}

For the efficient calculation of the TC probabilities $B_{z, r}^{T C}$, $B_{c}^{T C}$ (and consequently the total TC probability $B_{z, t o t}^{T C}=$ $B_{z, r}^{T C}+B_{c}^{T C}$ ) which refer to the proportion of time that there exist insufficient radio and computational RUs, respectively, we exploit the fact that the f-SC-MC model has a PFS and propose the following 3-step convolution algorithm:

Step 1

For each class $z$ of RRHs $(z=1, \ldots, Z)$ determine the occupancy distribution of each of the $M_{z}$ RRHs, $q_{\mathrm{fin}, z, m}(j)$, where $m=1, \ldots, M_{z}$ and $j=1, \ldots, C_{z}$, via:

$q_{\mathrm{fin}, z, m}(j)= \begin{cases}\left(\begin{array}{c}N_{z, m} \\ j\end{array}\right) \alpha_{z, m, \mathrm{idle}}^{j} q_{\mathrm{fin}, z, m}(0), & \text { for } j=1, \ldots, C_{z} \\ 0, & \text { for } j=C_{z}+1, \ldots, T\end{cases}$

The values of $q_{\mathrm{fin}, z, m}(j)$ can be normalized by the normalization constant $G_{\mathrm{fin}, z, m}=\sum_{j=0}^{C_{z}} q_{\mathrm{fin}, z, m}(j)$ and are denoted via $q_{\mathrm{fin}, z, m}^{\prime}(j)=q_{\mathrm{fin}, z, m}(j) / G_{\mathrm{fin}, z, m}$. Note that the first step of the proposed convolution algorithm treats each RRH individually from the rest RRHs. This treatment does not lead to exact TC probabilities unless the model has a PFS for the steady-state probabilities.

Step 2

Following the successive convolution of all RRHs (of all classes $Z$ ), apart from the $(z, 1) \mathrm{RRH}$, determine the aggregated occupancy distribution:

$$
\begin{aligned}
Q_{\mathrm{fin},(-(z, 1))}= & q_{\mathrm{fin}, 1,1}^{\prime} * \ldots * q_{\mathrm{fin}, 1, M_{1}}^{\prime} * \ldots * q_{\mathrm{fin}, z, 2}^{\prime} * \ldots \\
& * q_{\mathrm{fin}, z, M_{z}}^{\prime} * \ldots * q_{\mathrm{fin}, Z, 1}^{\prime} * \ldots * q_{\mathrm{fin}, Z, M_{Z}}^{\prime}
\end{aligned}
$$

The convolution operation between two occupancy distributions $q_{\mathrm{fin}, z, u}^{\prime}$ and $q_{\mathrm{fin}, z, w}^{\prime}$ is given by (13).

Step 3

Calculate the values of $B_{z \text { tot }}^{T C}$ based on the normalized values obtained from the convolution operation of step 2, as follows:

$$
B_{z, t o t}^{T C}=G_{\mathrm{fin}}^{-1}\left(q_{\mathrm{fin}, z, 1}^{\prime}\left(C_{z}\right) \sum_{r=0}^{T-C_{z}-1} Q_{\mathrm{fin},(-(z, 1))}^{\prime}(r)+q_{\mathrm{fin}}(T)\right)
$$

where: $q_{\mathrm{fin}, z, 1}^{\prime}\left(C_{z}\right)$ refers to the case of unavailable radio RUs in the $(z, 1)$ RRH (already determined in step 1), $Q_{\text {fin, },(-(z, 1))}^{\prime}(r)$ are the normalized values of $Q_{\text {fin, }(-(z, 1))}(r)$, while $q_{\text {fin }}(T)$ refers to the un-normalized probability of unavailable computational RUs, given by $q_{\mathrm{fin}}(T)=$ $\sum_{r=0}^{T} Q_{\mathrm{fin},(-(z, 1))}^{\prime}(r) \cdot q_{\mathrm{fin}, z, 1}^{\prime}(T-r)$ and $G$ is the normalization constant of the final convolution operation $Q_{\text {fin },(-(z, 1))}^{\prime} * q_{\text {fin }, z, 1}^{\prime}$ determined via (13).

As far as the CC probabilities for a call in the $m$-th RRH of class $z$ are concerned, they are determined for a system with $N_{z, m}-1$ traffic sources.

The occupancy distribution of the computational RUs can be obtained as follows:

$$
\begin{array}{ll}
q_{\mathrm{fin}}^{\prime}(0)=Q_{\mathrm{fin},(-(z, 1))}(0) \cdot q_{\mathrm{fin}, z, 1}^{\prime}(0) / G_{\mathrm{fin}}, & j=0 \\
q_{\mathrm{fin}}^{\prime}(j)=\sum_{r=0}^{j} Q_{\mathrm{fin},-(z, 1)}(r) \cdot q_{\mathrm{fin}, z, 1}^{\prime}(j-r) / G_{\mathrm{fin}}, & j=1, \ldots, T
\end{array}
$$

where: $G_{\text {fin }}$ is the normalization constant of the final convolution operation $Q_{\text {fin },(-(z, 1))} * q_{\text {fin }, z, 1}^{\prime}$.

Note that when $Z=1$, then all RRHs form a single cluster in the C-RAN and we have the f-SC-SC model. In that case, the interested reader may resort to [39] for the analysis of the model. Herein, we only present the corresponding 3 -step convolution algorithm for the calculation of the TC probabilities [39]:

\section{Step 1}

For each of the $M$ RRHs, calculate the occupancy distribution $q_{\text {fin }, m}(j)$, where $m=1, \ldots, M$ and $j=1, \ldots, C$, via:

$$
q_{\mathrm{fin}, m}(j)= \begin{cases}\left(\begin{array}{c}
N_{m} \\
j
\end{array}\right) \alpha_{m, \text { idle }}^{j} q_{\mathrm{fin}, m}(0), & \text { for } j=1, \ldots, C \\
0, & \text { for } j=C+1, \ldots, T\end{cases}
$$

The normalized values of $q_{\mathrm{fin}, m}(j)$ are expressed as $q_{\mathrm{fin}, m}^{\prime}(j)=q_{\mathrm{fin}, m}(j) / G_{\mathrm{fin}, m}$ where $G_{\mathrm{fin}, m}=\sum_{j=0}^{C} q_{\mathrm{fin}, m}(j)$.

Step 2

Following the successive convolution of all RRHs, apart from the 1st RRH, determine the aggregated occupancy distribution:

$$
Q_{\mathrm{fin},(-1)}=q_{\mathrm{fin}, 2}^{\prime} * \ldots * q_{\mathrm{fin}, m}^{\prime} * \ldots * q_{\mathrm{fin}, M}^{\prime}
$$

Taking into account two occupancy distributions $q_{\text {fin }, u}^{\prime}$ and $q_{\text {fin,w }}^{\prime}$, their convolution operation is given by (13). 


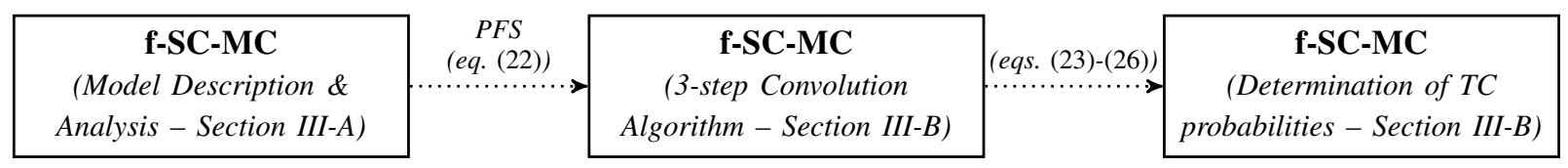

Fig. 3. The proposed methodology in the f-SC-MC model.

Step 3

Calculate the values of the total TC probability, $B_{t o t}^{T C}$, based on the normalized values obtainedin step 2 , as follows:

$$
B_{\text {tot }}^{T C}=B_{r}^{T C}+B_{c}^{T C}=G_{\text {fin }}^{-1}\left(q_{\text {fin }, 1}^{\prime}(C) \sum_{r=0}^{T-C-1} Q_{\text {fin },(-1)}(r)+q_{\text {fin }}(T)\right)
$$

where: $q_{\text {fin, } 1}^{\prime}(C)$ expresses the case where there are no radio RUs available in the 1 st RRH while $q_{\text {fin }}(T)$ refers to the (unnormalized) probability that there all computational RUs are unavailable, given by $q_{\mathrm{fin}}(T)=\sum_{x=0}^{T} Q_{\mathrm{fin},(-1)}(x) \cdot q_{\mathrm{fin}, 1}^{\prime}(T-$ $x)$ and $G_{\text {fin }}$ is the normalization constant of the convolution operation $Q_{\mathrm{fin},(-1)} * q_{\mathrm{fin}, 1}^{\prime}$ determined via (13).

The occupancy distribution of the computational RUs can be obtained as follows:

$$
\begin{array}{ll}
q_{\text {fin }}^{\prime}(0)=Q_{\text {fin },(-1)}(0) \cdot q_{\text {fin }, 1}^{\prime}(0) / G_{\text {fin }}, & j=0 \\
q_{\text {fin }}^{\prime}(j)=\sum_{r=0}^{j} Q_{\text {fin },(-1)}(r) \cdot q_{\text {fin }, 1}^{\prime}(j-r) / G_{\text {fin }}, & j=1, \ldots, T
\end{array}
$$

where: $G_{\text {fin }}$ is the normalization constant of the final convolution operation $Q_{\mathrm{fin},(-1)} * q_{\mathrm{fin}, 1}^{\prime}$.

Figure 3 summarizes the proposed methodology which is required in order to determine TC probabilities in the f-SC-MC model. Initially, it is important to have a PFS for the steadystate probabilities of the proposed model. Due to the fact that the model can be analyzed via a reversible Markov chain, we can obtain the PFS of (22). Based on the PFS, we can propose a convolution algorithm for the exact determination of TC probabilities.

\section{NUMERICAL RESUltS}

In this section, we present two application examples. In the first example, we provide analytical and simulation TC probabilities results of the f-SC-SC model of [39] and the corresponding analytical results of the SC-SC model of [33]. In the second example, we provide analytical and simulation TC probabilities results of the proposed f-SC-MC model and the corresponding analytical results of the SC-MC model of [38]. Simulation results are based on the Simscript III simulation language [40] and are mean values of 7 runs. In each run, two hundred million calls are generated while the first $5 \%$ of these generated calls are not considered in the TC probabilities results so as to account for a warm-up period. In addition, we do not present reliability ranges in the figures of this section, in order to increase their readability. These reliability ranges are less than two order of magnitude.

In the first example, consider a C-RAN of $M=6$ RRHs in the form of a single cluster. All RRHs have the same capacity $C=5$ radio RUs. On the other hand, the $\mathrm{V}$-BBU consist of $T$ computational RUs. We consider two values of $T: 1$ ) $T=M \cdot C=30$ and 2) $T=24$. Regarding the number of finite sources (or MUs) per RRH, we consider three values: a) $N=10$, b) $N=50$ and c) $N=100$ sources [39]. In each of the $M$ RRHs, let the offered traffic-load per idle MU be $\alpha_{m \text {,idle }}=\alpha_{m} / N$ where $\alpha_{m}$ expresses the value of the Poisson traffic. Contrary to the initial values of $\alpha_{m}$ chosen in [39] $\left(\alpha_{m}=2.0\right.$ erl for $\left.m=1, \ldots, 6\right)$, herein we choose $\alpha_{m}=$ 3.0 erl for $m=1, \ldots, 6$.

In the $\mathrm{x}$-axis of Figs. 4-7, the value of $\alpha_{m}$ increases in steps of 0.2 erl. So, point 1 is: $\left(\alpha_{1}, \alpha_{2}, \alpha_{3}, \alpha_{4}, \alpha_{5}, \alpha_{6}\right)=(3.0$, 3.0, 3.0, 3.0,3.0,3.0) while point 11 is: $\left(\alpha_{1}, \alpha_{2}, \alpha_{3}, \alpha_{4}, \alpha_{5}\right.$, $\left.\alpha_{6}\right)=(5.0,5.0,5.0,5.0,5.0,5.0)$. Contrary to the results presented in [39], the values of $\alpha_{m}$ chosen herein help us investigate an offered traffic-load region that leads to higher TC probabilities results (compare Figs. 3-6 of [39] with the corresponding figures of this example).

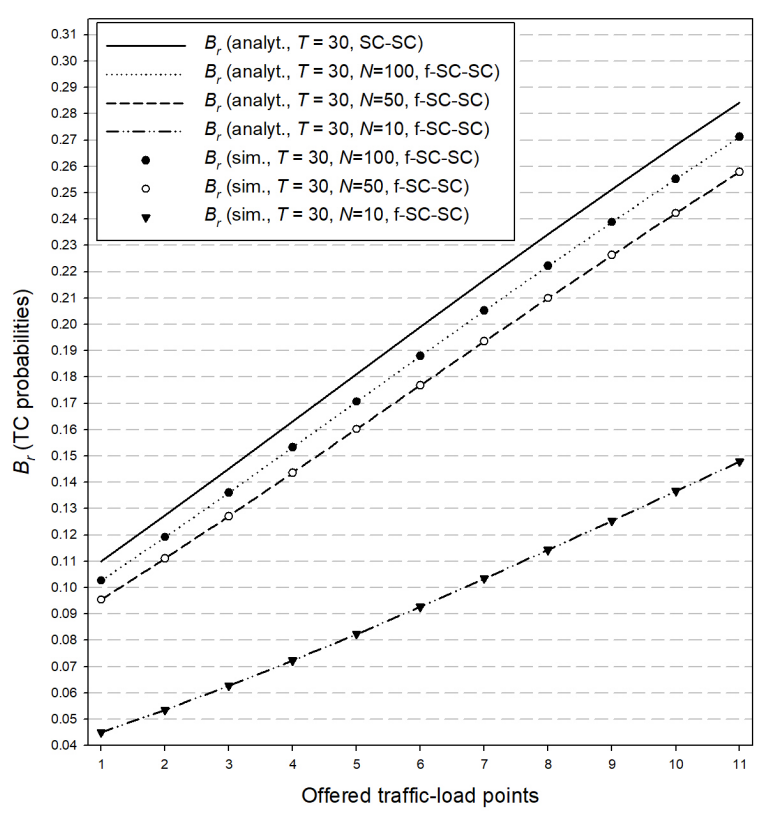

Fig. 4. TC probabilities $\left(B_{r}\right)$ for all values of $N$.

Figure 4 shows the simulation and analytical TC probabilities of $B_{r}$ for all values of $N$ as well as the corresponding TC probabilities for the SC-SC model of [33], assuming that $T=30$. Figure 5 presents the corresponding results of $B_{c}$. In both figures, we observe that: a) the existing SC-SC model leads to higher TC probabilities compared to those obtained via the f-SC-SC model of [39], especially when $N=10$ or 50 sources, b) increasing $N$ leads to TC probabilities which are closer to those obtained according to the SC-SC model (in which $N$ is infinite due to the Poisson call arrival process) and c) simulation results are very close to the analytical results. 


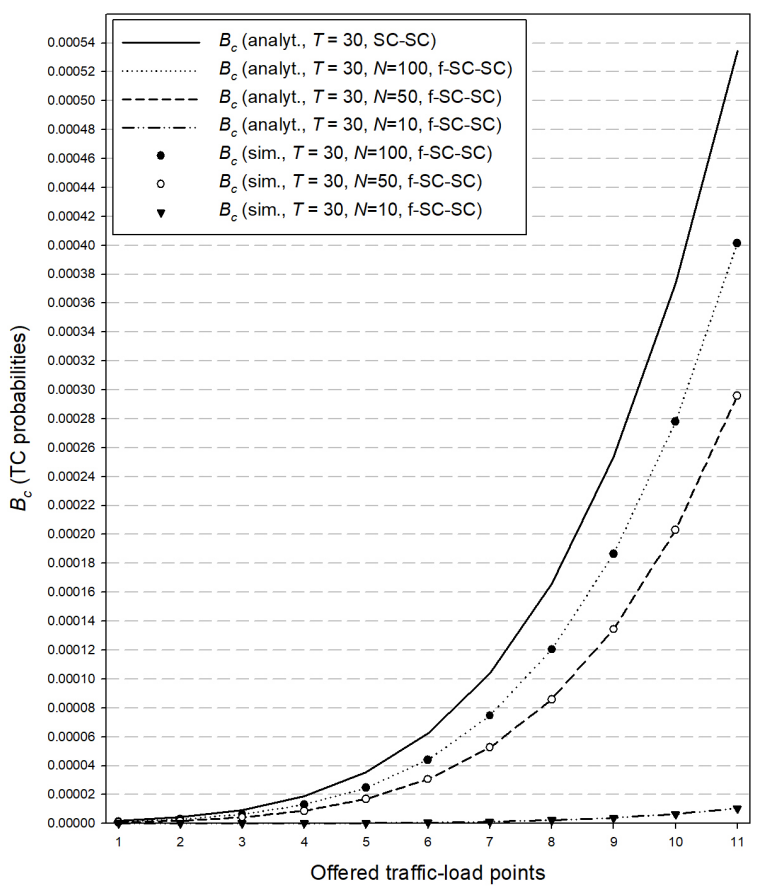

Fig. 5. TC probabilities $\left(B_{c}\right)$ for all values of $N$.

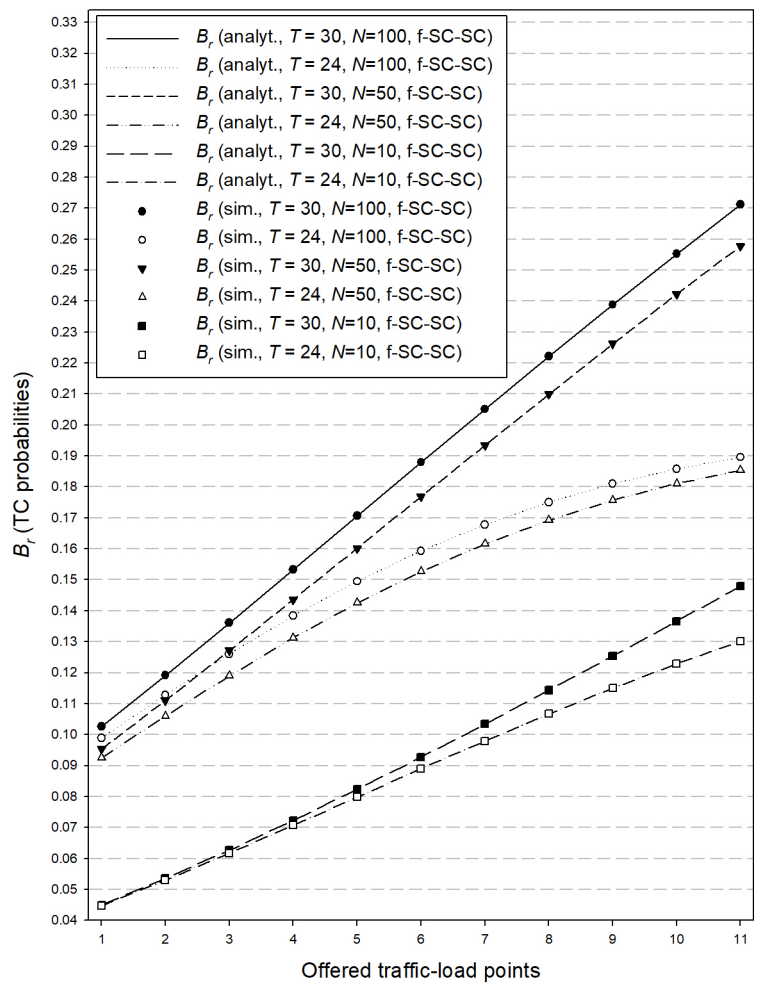

Fig. 6. TC probabilities $\left(B_{r}\right)$ for all values of $N$ and $T$.

Figure 6 presents the simulation and analytical TC probabilities of $B_{r}$ for all values of $N$ and both values of $T$. We observe that a decrease in $T$ results in a decrease of $B_{r}$ especially for high values of the offered traffic-load. This behavior can be explained as follows: a decrease in $T$ leads many new calls to blocking due to the unavailability of computational RUs, and therefore the number of available

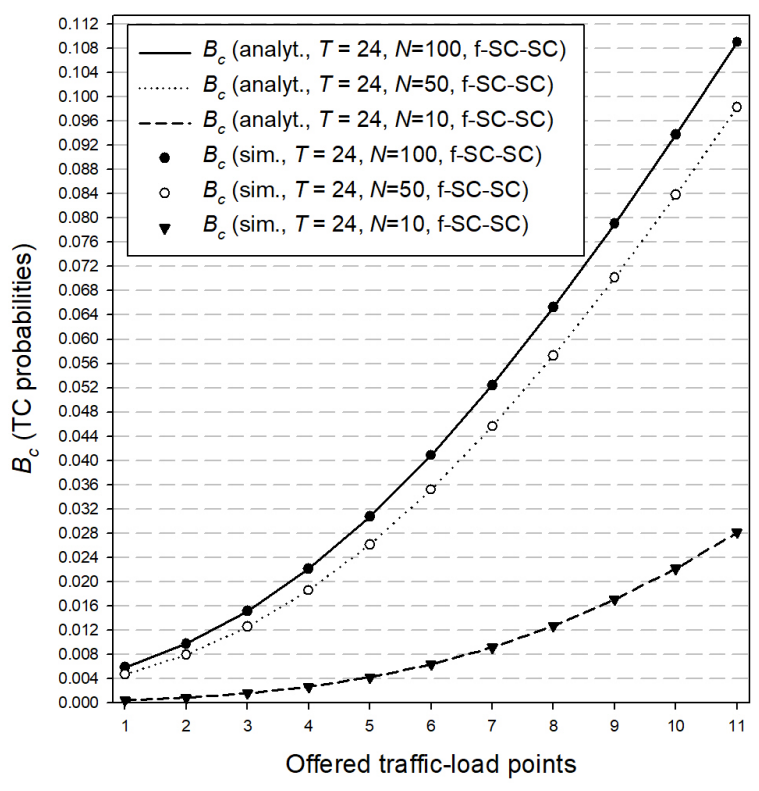

Fig. 7. TC probabilities $\left(B_{c}\right)$ for all values of $N$ and $T=24$.

radio RUs increases.

Figure 7 presents the simulation and analytical TC probabilities of $B_{c}$ for all values of $N$ and $T=24$. We observe that the decrease of $T$ to 24 units, results in a remarkable increase of $B_{c}$ (compare Figs. 5 and 7) which reveals that particular attention is required when choosing the value of $T$ especially if it is much lower than $M \cdot C$.

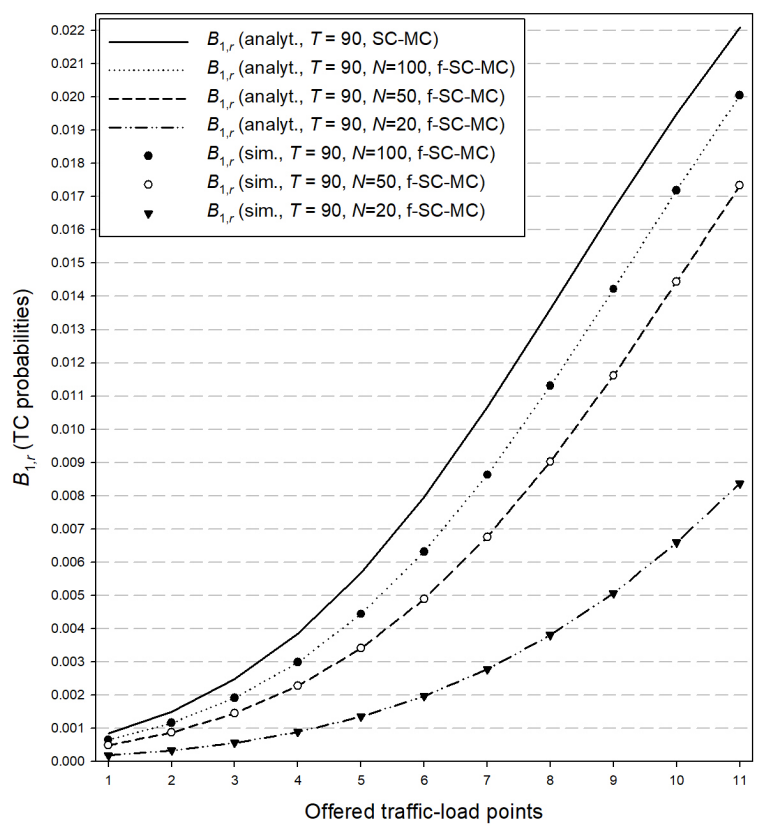

Fig. 8. TC probabilities $\left(B_{1, r}\right)$ for different values of $N$ (class $z=1$ ).

In the second example, consider a C-RAN of $Z=3$ classes of RRHs. The first class includes $M_{1}=10$ RRHs of capacity $C_{1}=8$ radio RUs. The second class includes $M_{2}=5$ RRHs of capacity $C_{2}=12$ radio RUs. Finally, the third class includes $M_{3}=2$ RRHs of capacity $C_{3}=15$ radio RUs. The V-BBU consists of $T=90$ computational RUs. Regarding the number 
of finite sources for the $m$-th RRH (of each class $z=1,2,3$ ), we consider the values: a) $N_{z, m}=20$, b) $N_{z, m}=50$ and c) $N_{z . m}=100$ sources. The offered traffic-load per idle MU in each of the $M$ RRHs is $\alpha_{z, m \text {,idle }}=\alpha_{z} / N_{z, m}$ where $\alpha_{z}$ refers to the value of the Poisson traffic. Initially, we assume that $\alpha_{1}=2.0$ erl for each RRH of the first class $(m=1, \ldots, 10)$. Similarly, let $\alpha_{2}=4.0$ erl for each RRH of the second class ( $m=1, \ldots, 5)$ and $\alpha_{3}=6.0$ erl for each RRH of the third class $(m=1,2)$.

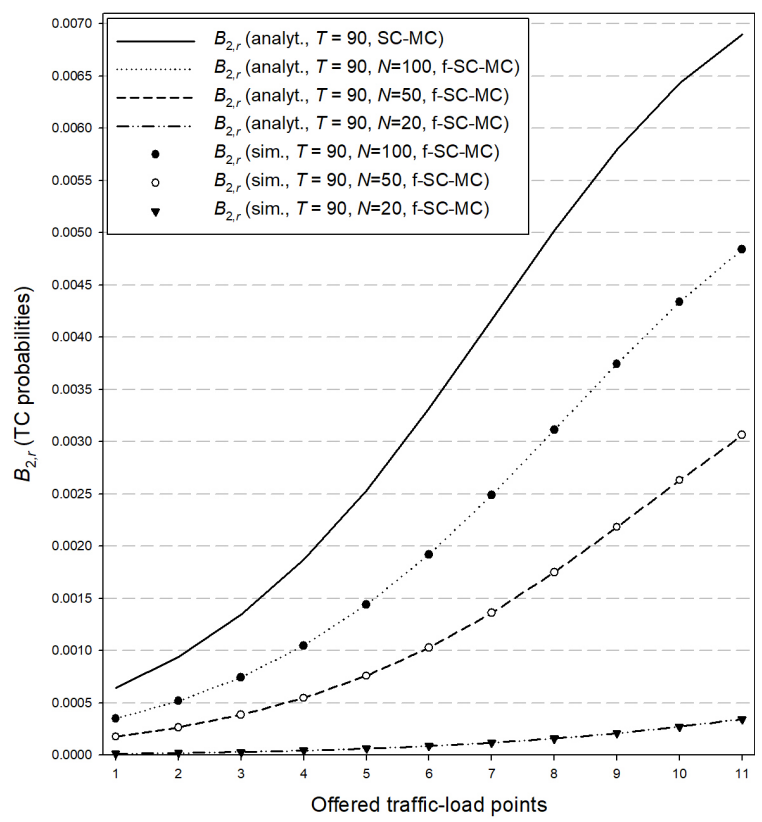

Fig. 9. TC probabilities $\left(B_{2, r}\right)$ for different values of $N$ (class $z=2$ ).

In the $\mathrm{x}$-axis of Figs. 8-10, the value of $\alpha_{z}$ increases in steps of 0.2 erl. So, point 1 is: $\left(\alpha_{1}, \alpha_{2}, \alpha_{3}\right)=(2.0,4.0,6.0)$ while point 11 is: $\left(\alpha_{1}, \alpha_{2}, \alpha_{3}\right)=(4.0,6.0,8.0)$.

Figures 8-10 present the analytical and simulation TC probabilities of $B_{z, r}^{T C}$ (depicted for presentation purposes as $B_{z, r}$ ) for each class $z$ and the three values of $N_{z, m}$ (depicted for presentation purposes as $N$ ). For comparison, we also include the corresponding TC probabilities for the SC-MC of [38]. In all figures, we observe that: a) the existing SCMC model provides much higher TC probabilities compared to those obtained via the f-SC-MC model, especially when the values of $N$ are low (e.g., when $N=20$ or 50 sources), b) increasing $N$ results in TC probabilities which are closer to those obtained via the SC-MC model (where by definition $N$ is infinite) and c) simulation results are very close to the analytical results

\section{CONCLUSION}

In this paper we propose a new loss model, named f-SC-MC model, for the analysis of a C-RAN that accommodates quasirandom traffic which is smoother than the classical Poisson process considered in [33], [38]. Two different cases are considered regarding the grouping of RRHs. The single cluster case, proposed in [39] and the multi-cluster case proposed

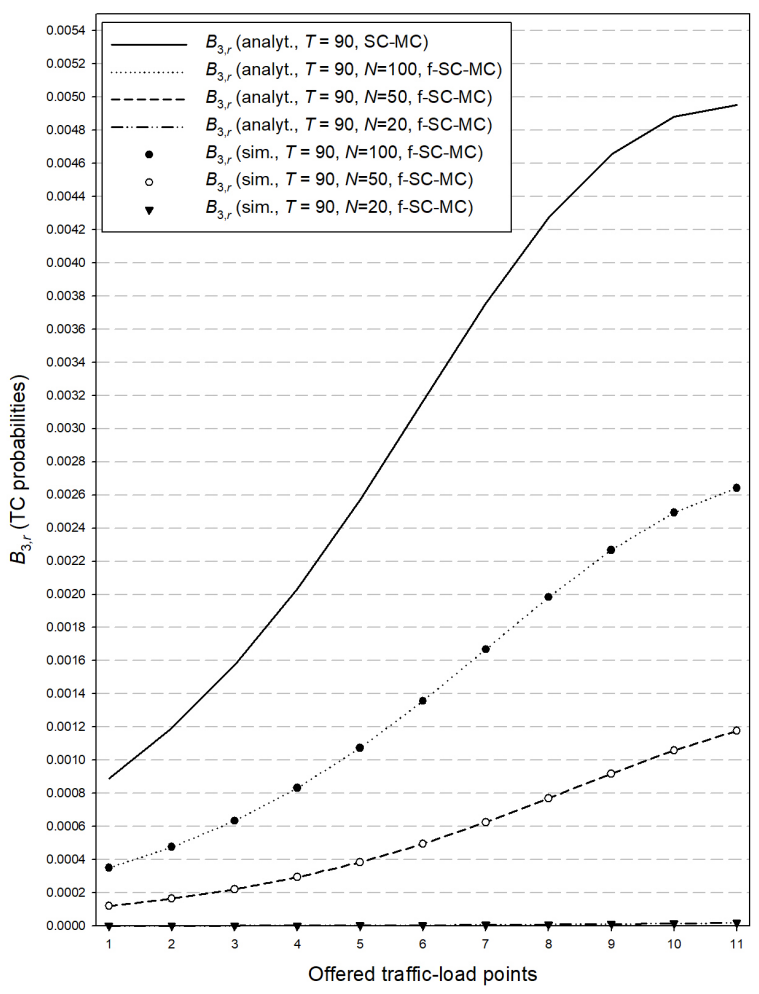

Fig. 10. TC probabilities $\left(B_{3, r}\right)$ for different values of $N$ (class $z=3$ ).

herein. The latter generalizes the model of [39] since it assumes that RRHs are grouped in different clusters according to their capacities in radio RUs. Our first major contribution is to show that the steady-state probabilities of the proposed model in the multi-cluster case (f-SC-MC model) have a PFS. This is an essential step in order to proceed with our second major contribution which is a convolution algorithm for the exact determination of TC probabilities in the f-SCMC model. Indeed, the first step of the proposed convolution algorithm treats each RRH individually from the rest RRHs. This treatment does not lead to exact TC probabilities unless the model has a PFS for the steady-state probabilities. The accuracy of the proposed algorithm is verified via simulation and found to be very satisfactory. As a future work we intend to study call arrival processes that are burstier than the Poisson process such as the batched Poisson process [41]-[44].

\section{REFERENCES}

[1] M. Habibi, M. Nasimi, B. Han, and H. Schotten, "A comprehensive survey of RAN architectures toward 5G mobile communication system", IEEE Access, pp. 70371-70421, May 2019. doi: 10.1109/ACCESS.2019.2919657

[2] M. Hossain, A. Mahin, T. Debnath, F. Mosharrof, and K. Ziaul, "Recent research in cloud radio access network (C-RAN) for 5G cellular systems - A survey", Journal Netw. Comp. Applic., vol. 139, pp. 31-48, Aug. 2019. doi: 10.1016/j.jnca.2019.04.019

[3] A. Checko et al., "Cloud RAN for mobile networks - A technology overview", IEEE Commun. Surveys Tuts., vol. 17, no. 1, pp. 405-426, 1st Quart., 2015. doi: 10.1109/COMST.2014.235525

[4] CPRI Consortium, "CPRI Specification V7.0 Common Public Radio Interface (CPRI); Interface Specification", 2015.

[5] ETSI GS NFV-MAN 001 (V1.1.1), "Network Function Virtualisation $(N F V)$; Management and Orchestration”, Dec. 2014. 
[6] M. Stasiak, M. Glabowski, A. Wisniewski, and P. Zwierzykowski, Modeling and dimensioning of mobile networks. John Wiley, Hoboken, NJ, USA, 2011. doi:10.1002/9780470976036

[7] I. Moscholios and M. Logothetis, Efficient multirate teletraffic loss models beyond Erlang. John Wiley \& IEEE Press, Hoboken, NJ, USA, 2019. doi:10.1002/9781119426974

[8] A. Kaliszan, M. Glabowski, and M. Stasiak, "Generalized convolution algorithm for modelling state dependent systems", IET Circuits Devices Syst., vol. 8, issue 5, pp. 378-386, Sept. 2014. doi: 10.1049/ietcds. 2013.0479

[9] S. Hanczewski, A. Kaliszan, and M. Stasiak, "Convolution model of a queueing system with the cFIFO service discipline", Mobile Inform. Syst., volume 2016, Article ID 2185714, 15 pages. doi: $10.1155 / 2016 / 2185714$

[10] A. Kaliszan and M. Stasiak, "The possibilities and limitations of the application of the convolution algorithms for modeling network systems", in Proc. 24th Int. Conf. on Computer Networks, Ladek Zdroj, Poland, June 2017. doi: 10.1007/978-3-319-59767-6 18

[11] I. Moscholios, V. Vassilakis, G. Bouloukakis, P. Panagoulias, and M. Logothetis, "A convolution algorithm for a multirate loss system with Poisson arrivals and a threshold call admission policy", in Proc. IEEE/IET CSNDSP, Budapest, Hungary, July 2018. doi: 10.1109/CSNDSP.2018.8471771

[12] M. Glabowski, A. Kaliszan, and M. Stasiak, "Asymmetric convolution algorithm for full-availability group with bandwidth reservation", in Proc. Asia-Pacific Conf. on Communications, Busan, South Korea, Sept. 2006. doi: 10.1109/APCC.2006.255768

[13] M. Glabowski, A. Kaliszan, and M. Stasiak, "Asymmetric convolution algorithm for blocking probability calculation in full-availability group with bandwidth reservation", IET Circuits Devices Syst., vol. 2, issue 1, pp. 87--94, Feb. 2008. doi: 10.1049/iet-cds:20070037

[14] Q. Huang, K. Ko, and V. Iversen, "A new convolution algorithm for loss probability analysis in multiservice networks", Perf. Eval., vol. 68, issue 1, pp. 76-87, Jan. 2011. doi: 10.1016/j.peva.2010.09.007

[15] S. Sagkriotis and I. Moscholios, "Evaluation of convolution algorithms in the Erlang multirate loss model under the bandwidth reservation policy", in Proc. IEICE ICTF, Poznan, Poland, July 2017.

[16] S. Sagkriotis, S. Pantelis, I. Moscholios, and V. Vassilakis, "Call blocking probabilities in a two-link multi rate loss system for Poisson traffic", IET Netw., vol. 7, issue 4, pp. 233-241, July 2018. doi: ietnet. 2017.0223

[17] M. Glabowski, A. Kaliszan, and M. Stasiak, "On the application of the asymmetric convolution algorithm in modeling of full-availability group with bandwidth reservation", in Proc. Int. Teletraffic Congress, Ottawa Canada, June 2007. doi: 10.1007/978-3-540-72990-7_76

[18] M. Glabowski, A. Kaliszan, and M. Stasiak, "Convolution algorithm for state-passage probabilities calculation in limited availability group", in Proc. AICT, Athens, Greece, June 2008. doi: 10.1109/AICT.2008.45

[19] J. Vardakas, I. Moscholios, M. Logothetis, and V. Stylianakis, "An analytical approach for dynamic wavelength allocation in WDMTDMA PONs servicing ON-OFF traffic", IEEE/OSA Journal of Optical Commun. Netw., vol. 3, issue 4, pp. 347-358, April 2011. doi 10.1364/JOCN.3.000347

[20] I. Moscholios, J. Vardakas, M. Logothetis, and A. Boucouvalas, "Congestion probabilities in a batched Poisson multirate loss model supporting elastic and adaptive traffic", Annals Telecommun., vol. 68, issue 5 , pp. 327-344, June 2013. doi: 10.1007/s12243-012-0326-7

[21] J. Vardakas, I. Moscholios, M. Logothetis, and V. Stylianakis, "Performance analysis of OCDMA PONs supporting multi-rate bursty traffic", IEEE Trans. Commun., vol. 61, no. 8, pp. 3374-3384, Aug. 2013. doi: 10.1109/TCOMM.2013.061913.120798

[22] A. Kaliszan, M. Glabowski, and M. Stasiak, "Optimized two dimensional convolution algorithm for modelling systems with overflow traffic", in Proc. IEEE-IET CSNDSP, Manchester, U.K, July 2014. doi: 10.1109/CSNDSP.2014.6923787

[23] I. Moscholios, M. Logothetis, J. Vardakas, and A. Boucouvalas, "Performance metrics of a multirate resource sharing teletraffic mode with finite sources under both the threshold and bandwidth reservation policies", IET Netw., vol. 4, issue 3, pp. 195-208, May 2015. doi: 10.1049/iet-net.2014.0050

[24] I. Moscholios, V. Vassilakis, M. Logothetis, and A. Boucouvalas, "A probabilistic threshold-based bandwidth sharing policy for wireless multirate loss networks", IEEE Wireless Commun. Letters, vol. 5, issue 3, pp 304-307, June 2016. doi: 10.1109/LWC.2016.2547913

[25] L. Wang and S. Zhou, "On the fronthaul statistical multiplexing gain", IEEE Commun. Letters, vol. 21, issue 5, pp. 1099-1102, May 2017. doi: 10.1109/LCOMM.2017.2653120
[26] L. Larsen, A. Checko, and H. Christiansen, "A survey of the functional splits proposed for 5G mobile crosshaul networks", IEEE Commun. Surveys Tuts, vol 21, issue 1, pp.146-172, 1st Quart., 2019. doi: 10.1109/COMST.2018.2868805

[27] B. Dai and W. Yu, "Energy efficiency of downlink transmission strategies for cloud radio access networks", IEEE Journal Selected Areas Commun., vol. 34, issue 4, pp. 1037-1050, April 2016. doi: 10.1109/JSAC.2016.2544459

[28] Y. Li, T. Jiang, K. Luo, and S. Mao, "Green heterogeneous cloud radio access networks: potential techniques, performance trade-offs, and challenges", IEEE Commun. Mag., vol. 55, issue 11, pp. 33-39, Nov. 2017. doi: 10.1109/MCOM.2017.1600807

[29] I. Ahmad et al., "Overview of 5G security challenges and solutions", IEEE Commun. Standards Mag., vol. 2, issue 1, pp. 36-43, March 2018. doi: 10.1109/MCOMSTD.2018.1700063

[30] W. Ejaz, S. Sharma, S. Saadat, M. Naeem, A. Anpalagan, and N. Chughtai, "A comprehensive survey on resource allocation for CRAN in 5G and beyond networks", accepted for publication in Journal Netw. Comp. Applic., March 2020. doi: 10.1016/j.jnca.2020.102638

[31] V. Quintuna-Rodriguez and F. Guillemin, "On dimensioning cloud-ran systems", in Proc. 11th EAI Int. Conf. on Perf. Eval. Methodologies and Tools, Venice, Italy, Dec. 2017. doi: 10.1145/3150928.3150937

[32] V. Quintuna-Rodriguez and F. Guillemin, "Cloud-RAN modeling based on parallel processing," IEEE Journal Selected Areas Commun., vol. 36, issue 3, pp. 457-468, March 2018. doi: 10.1109/JSAC.2018.2815378

[33] J. Liu, S. Zhou, J. Gong, Z. Niu, and S. Xu, "On the statistical multiplexing gain of virtual base station pools", in Proc. IEEE Globe com, Austin, TX, USA, Dec. 2014, pp. 2283-2288. doi: 10.1109/GLOCOM.2014.7037148

[34] A. Avramova, H. Christiansen, and V. Iversen, "Cell deployment optimization for cloud radio access network using teletraffic theory", in Proc. Advanced Int. Conf. Telecommun. (AICT), Brussels, Belgium, June 2015.

[35] A. Checko, A. Avramova, M. Burger, and H. Christiansen, "Evaluating C-RAN fronthaul functional splits in terms of network level energy and cost savings", Journal Commun. Networks, vol. 18, no. 2, pp. 162-172, April 2016. doi: 10.1109/JCN.2016.000025

[36] V. Iversen, V. Benetis, and P. Hansen, "Performance of hierarchical cellular networks with overlapping cells", in Proc. EuroNGI Workshop, Wadern, Germany, June 2004. doi: 10.1007/978-3-540-31963-4_2

[37] I. Moscholios, V. Vassilakis, M. Logothetis, and A. Boucouvalas, "State-dependent bandwidth sharing policies for wireless multirate loss networks", IEEE Trans. Wireless Commun., vol. 16, issue 8, pp. 54815497, Aug. 2017. doi: 10.1109/TWC.2017.2712153

[38] J. Liu, S. Zhou, J. Gong, Z. Niu, and S. Xu, "Statistical multiplexing gain analysis of heterogeneous virtual base station pools in cloud radio access networks", IEEE Trans. Wireless Commun., vol. 15, no. 8, pp. 5681--5694, Aug. 2016. doi: 10.1109/TWC.2016.2567383

[39] I-A. Chousainov, I. Moscholios, A. Kaloxylos, and M. Logothetis, "Performance evaluation of a C-RAN supporting quasi-random traffic", in Proc. IEEE Softcom, Split, Croatia, Sept. 2019. doi: 10.23919/SOFTCOM.2019.8903712

[40] Simscript III http://www.simscript.com/.(accessed on April. 2020)

[41] I. Moscholios and M. Logothetis, "The Erlang multirate loss model with batched Poisson arrival processes under the bandwidth reservation policy", Comp. Commun., vol. 33, supplement 1, pp. S167-S179, Nov. 2010. doi: 10.1016/j.comcom.2010.04.039

[42] I. Moscholios, J. Vardakas, M. Logothetis, and A. Boucouvalas, "QoS guarantee in a batched Poisson multirate loss Model supporting elastic and adaptive traffic", in Proc. IEEE ICC, Ottawa, Canada, 2012. doi 10.1109/ICC.2012.6363934

[43] P. Ezhilchelvan and I. Mitrani, "Multi-class resource sharing with batch arrivals and complete blocking", in Proc. Int. Conf. on Quantitative Evaluation of Systems (QEST), Lecture Notes in Computer Science, Vol. 10503, Springer, 2017. doi: 10.1007/978-3-319-66335-7_10

[44] I. Moscholios, V. Vassilakis, and P. Sarigiannidis, "Performance modelling of a multirate loss system with batched Poisson arrivals under probabilistic threshold policy", IET Netw., vol. 7, issue 4, pp. 242-247, July 2018. doi: 10.1049/iet-net.2017.0216 


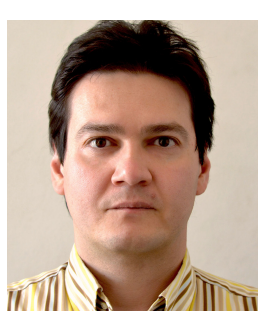

Iskanter-Alexandros Chousainov received the B.S. degree from the Dept. of Mathematics of the University of the Aegean in 2002, the M.Sc degree in Information Systems from Hellenic Open University in 2017. He is currently a Ph.D. student at the Dept. of Informatics \& Telecommunications, University of Peloponnese, Tripolis, Greece. His research interests include teletraffic engineering and performance evaluation of $5 \mathrm{G}$ networks.

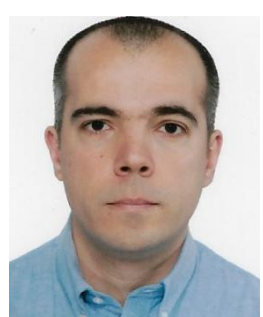

Ioannis D. Moscholios was born in Athens, Greece, in 1976. He received the Dipl.-Eng. degree in Electrical \& Computer Engineering from the University of Patras, Patras, Greece, in 1999, the M.Sc. degree in Spacecraft Technology \& Satellite Communications from the University College London, UK, in 2000 and the Ph.D. degree in Electrical \& Computer Engineering from the University of Patras, in 2005 From 2005 to 2009 he was a Research Associate at the Wire Communications Laboratory, Dept. of Electrical \& Computer Engineering, University of Patras. From 2009 to 2013 he was a Lecturer in the Dept. of Telecommunications Science and Technology, University of Peloponnese, Tripolis, Greece. From 2013 to 2018 he was an Assistant Professor in the Dept. of Informatics \& Telecommunications, University of Peloponnese, Tripolis, Greece. Currently, he is an Associate Professor in the Dept. of Informatics \& Telecommunications, University of Peloponnese, Tripolis, Greece. His research interests include teletraffic engineering, simulation and performance analysis of communication networks. He has published over 170 papers in international journals/ conferences and is a co-author of the book: Efficient Multirate Teletraffic Loss Models Beyond Erlang (IEEE Press, Wiley, April 2019). He has served as a Guest Editor in: a) IET Communications, b) IET Networks, c) Applied Sciences and d) Mobile Information Systems. He has also served as an Associate Editor in IEICE Transactions on Communications. $\mathrm{He}$ is an IARIA Fellow and a member of the Technical Chamber of Greece (TEE).

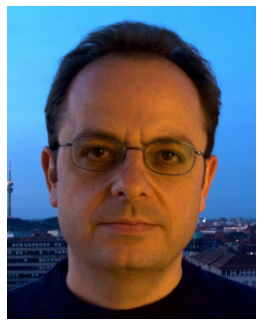

Alexandros Kaloxylos (B.Sc., M.Phil., Ph.D.) received his Ph.D. degree in Informatics and Telecommunications from the University of Athens in 1999. Since 1994, he has participated, as a researcher, in numerous projects realized in the context of EU Programmes as well as national initiatives. In 2002 he joined the faculty of the University of Peloponnese, where he is currently an Associate Professor in the Department of Informatics and Telecommunications. From 2014 until 2017, he worked as a Principal Researcher in Huawei's European Research Center in Munich, where he led the Radio Access Network team in the design of 5G networks. During 2016, he was the vice-chair of the 5G PPP Architecture WG. Since October 2019, he is the Technology Board Chairman of 5G-PPP. $\mathrm{He}$ has published over 130 papers in international journals, conferences and book chapters and has filed several patents for topics related to $5 \mathrm{G}$ networks.

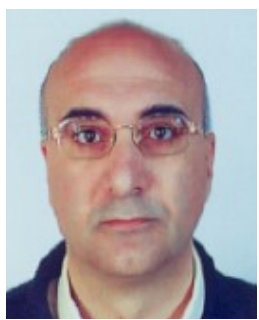

Michael D. Logothetis (SM'08) received the Dipl.Eng. and Ph.D. degrees in electrical engineering from the University of Patras, Patras, Greece, in 1981 and 1990, respectively. From 1982 to 1990 , he was a Teaching and Research Assistant with the Laboratory of Wire Communications, University of Patras. He has participated in many national and EU research programs, dealing with telecommunication networks and office automation. From 1991 to 1992, he was Research Associate with NTT's Telecommunication Networks Laboratories, Tokyo, Japan. He was a Lecturer with the Electrical and Computer Engineering Department, University of Patras, and in 2009 was elected a (Full) Professor in the same department. He has authored over 220 conference/journal papers and is a co-author of the book: Efficient Multirate Teletraffic Loss Models Beyond Erlang (IEEE Press, Wiley, April 2019). His current research interests include teletraffic theory and engineering, traffic/network control, simulation and performance optimization of telecommunications networks. He organized (was General Chair) of the IEEE/IET CSNDSP 2006 and IEICE ICTF 2016 He has served as a TPC member of several international conferences while he has chaired many technical sessions. He was a Guest Editor of five journals: Mediterranean Journal of Electronics and Communications, Mediterranean Journal of Computers and Networks, IET Circuits, Devices \& Systems, IET Networks, and Ubiquitous Computing and Communication Journal. He is a senior member of IEEE, a member of the IARIA (Fellow), the IEICE, the FITCE, and the Technical Chamber of Greece. 